\title{
A Blue-pigmented Lesion on the Cheek in a Three-year-old Girl: A Quiz
}

Charlotte MOREAU ${ }^{1,2}$, Anne LE TOUZE ${ }^{3}$, Fanny DUJARDIN ${ }^{4}$ and Annabel MARUANI ${ }^{1,2}$

${ }^{1}$ University of Tours, ${ }^{2}$ Department of Dermatology, Unit of Paediatric Dermatology, CHRU Tours, FR-37000 Tours, ${ }^{3}$ Department of Paediatric Surgery and ${ }^{4}$ Department of Pathology CHRU Tours, Tours, France. E-mail: charlotte.moreau29@hotmail.fr

A 3-year-old girl with no medical history was referred to the department of paediatric surgery for a pigmented lesion on the right cheek (Fig. 1a). Her parents reported that the lesion had appeared during the first 6 months of life and had increased in size progressively with the child's growth.

Physical examination revealed a dark-blue macule 6 $\mathrm{mm}$ in diameter. The examination was otherwise normal. Dermoscopy was not conducted.

The lesion was removed under local anaesthesia. Microscopy examination showed intense dermal proliferation of melanocytes, outnumbered by melanophages,
$1.6 \mathrm{~mm}$ in thickness (Fig. 1b). The tumour contained a high number of epithelioid cells with large nuclei and prominent nucleoli. The pigmented cells were clustered or spindled (Fig. 1c). Immunohistochemistry revealed intense immunostaining for MelanA and human melanoma black (HMB) 45 (Fig. 1d), which confirmed the melanocytic origin of the tumour. There was limited expression of p16 and loss of expression of protein kinase A regulatory subunit- $\alpha$ (PRKAR1A).

What is your diagnosis? See next page for answer.

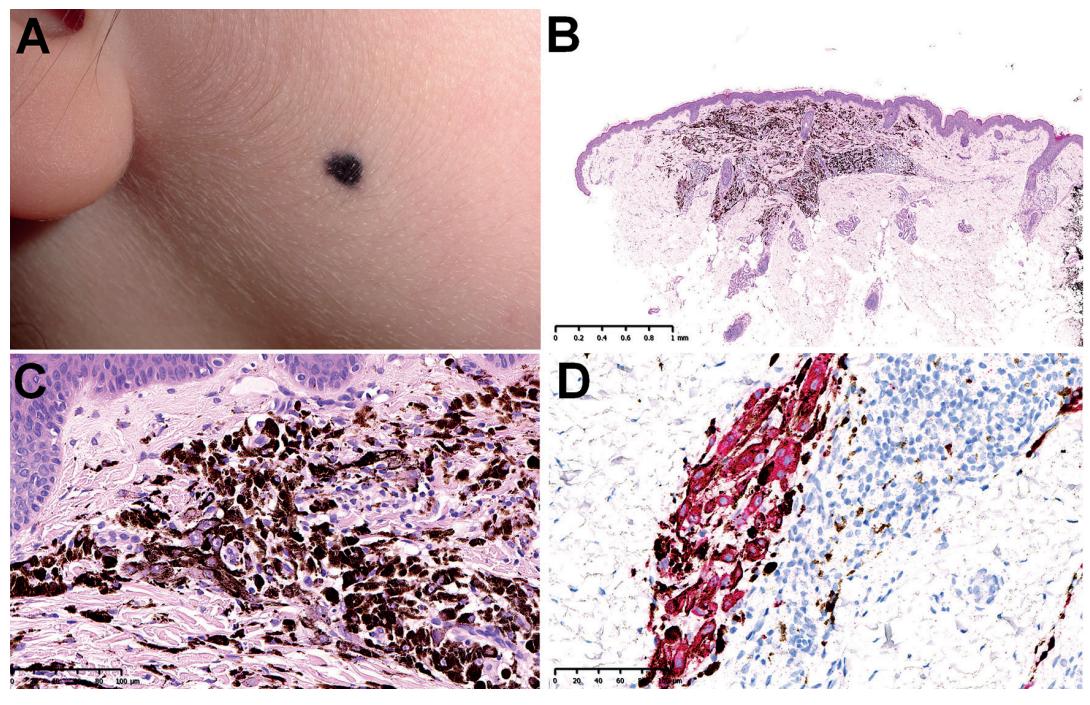

Fig. 1. (a) Dark-blue macule on the right cheek of a 3-year-old girl. (b) Microscopy examination $(\times 2)$ with haematoxylin and eosin staining showing a dermal proliferation of melanocytes and numerous melanophages. (c) Microscopy $(\times 20)$ with haematoxylin and eosin staining showing epithelioid and pigmented cells clustered and spindled. (d) Immunohistochemistry with intense staining for HMB 45. 


\section{ANSWERS TO QUIZ}

\section{A Blue-pigmented Lesion on the Cheek in a Three- year-old Girl: A Commentary}

Acta Derm Venereol 2021; 101: adv00580.

\section{Diagnosis: Pigmented epithelioid melanocytoma}

Pigmented epithelioid melanocytoma (PEM) is also known as "animal-type melanoma". This entity was initially described in 1832 in veterinary medicine, in horses (1). The term "pigmented epithelioid melanocytoma" further emerged to describe the spectrum of borderline tumours ranging from an epithelioid blue naevus to animal-type melanoma (2-4).

PEM belongs to melanocytic tumours of uncertain malignant potential, as in atypical Spitz naevus; for example, because classifying them as benign or malignant tumours is difficult, both from a clinical and histological point of view, and the prognosis is unpredictable (5).

PEM can occur at all ages, with cases described in newborns as well as older people. No sex predominance has been evidenced (6). In most cases, the tumour presents as a black or dark-blue papule, showing progressive growth. There are very few descriptions of dermoscopic features of this tumour in the literature, but these mainly report homogeneous blue pigmentation, inconstantly associated with black coloured areas or white crystalline structures. Dermoscopically, they might be confused with blue naevus or melanoma (7).

More rarely, PEM can have clinical features of an atypical Spitzoid lesion or hyperpigmented atypical naevus (8). Both are differential diagnoses for PEM, as are blue naevus, deep penetrating naevus, adnexal tumours, and melanoma (6). In rare cases, PEM might be associated with Carney syndrome, a rare, autosomal dominant, multiple endocrine neoplasia, and lentiginosis syndrome (9). There were no signs of such syndrome in the current patient.

Microscopy examination of a PEM shows a proliferation of heavily pigmented epithelioid and spindled melanocytes. These are characterized by inactivation of PRKAR1A protein. The tumour usually features a low mitotic index, low cellularity, rare ulcerations, and no regression or vascular invasion $(3,4)$.

Although PEM is considered more indolent than conventional melanoma (10), it seems to have a propensity for regional lymphatic metastasis, with, in contrast, exceptional distant metastasis. Young patients seem to have better prognosis than older patients $(2,3)$. A review of the literature from 2015 including 190 cases showed a rate of lymph-node metastasis of $41.0 \%$, local recurrence $7.6 \%$, and distant metastasis $3.0 \%$ (6). The median follow-up in these studies was 36 months. In publications of animals with a long-term follow-up, the lesions are described as having first a long indolent period, followed by a more aggressive late phase (3).

The management of PEM lacks guidelines and consensus. Most authors recommend revision surgery with safety margins as in conventional melanomas, and regular physical follow-up, with or without lymphatic ultrasonography (6). In the current case, after multidisciplinary consultation, it was decided to perform revision surgery with 5-mm margins and 6-month follow-up for 3 years. There was no recurrence after a follow-up of 24 months.

\section{REFERENCES}

1. Dick W. Melanosis in men and horses. The Lancet 1832; 19: 192

2. Zembowicz A, Carney JA, Mihm MC. Pigmented epithelioid melanocytoma: a low-grade melanocytic tumor with metastatic potential indistinguishable from animal-type melanoma and epithelioid blue nevus. Am J Surg Pathol 2004; 28: $31-40$.

3. Ludgate MW, Fullen DR, Lee J, Rees R, Sabel MS, Wong SL, et al. Animal-type melanoma: a clinical and histopathological study of 22 cases from a single institution. Br J Dermatol 2010; 162: 129-136.

4. Cohen JN, Yeh I, Mully TW, LeBoit PE, McCalmont TH. Genomic and clinicopathologic characteristics of PRKAR1Ainactivated melanomas: toward genetic distinctions of animal-type melanoma/pigment synthesizing melanoma. Am J Surg Pathol 2020; 44: 805-816.

5. de la Fouchardiere A, Blokx W, van Kempen LC, Luzar B, Piperno-Neumann S, Puig S, et al. ESP, EORTC, and EURACAN Expert Opinion: practical recommendations for the pathological diagnosis and clinical management of intermediate melanocytic tumors and rare related melanoma variants. Virchows Arch 2021; 479: 3-11.

6. Vyas R, Keller JJ, Honda K, Cooper KD, Gerstenblith MR. A systematic review and meta-analysis of animal-type melanoma. J Am Acad Dermatol 2015; 73: 1031-1039.

7. Moscarella E, Ricci R, Argenziano G, Lallas A, Longo C, Lombardi $M$, et al. Pigmented epithelioid melanocytoma: clinical, dermoscopic and histopathological features. Br J Dermatol 2016; 174: 1115-1117.

8. Isales MC, Mohan LS, Quan VL, Garfield EM, Zhang B, Shi $\mathrm{K}$, et al. Distinct genomic patterns in pigmented epithelioid melanocytoma: a molecular and histologic analysis of 16 cases. Am J Surg Pathol 2019; 43: 480-488.

9. Carney JA, Ferreiro JA. The epithelioid blue nevus. A multicentric familial tumor with important associations, including cardiac myxoma and psammomatous melanotic schwannoma. Am J Surg Pathol 1996; 20: 259-272.

10. Antony FC, Sanclemente G, Shaikh H, Trelles AS, Calonje E. Pigment synthesizing melanoma (so-called animal type melanoma): a clinicopathological study of 14 cases of a poorly known distinctive variant of melanoma. Histopathology 2006; 48: 754-762. 